\title{
Erratum: On a new type of divergence for spiky Wilson loops and related entanglement entropies
}

\author{
Harald Dorn \\ Institut für Physik und IRIS Adlershof, Humboldt-Universität zu Berlin, \\ Zum Großen Windkanal 6, D-12489 Berlin, Germany \\ E-mail: dorn@physik.hu-berlin.de
}

ERRATUM TO: JHEP03(2018)124

ARXiv EPrint: 1801.10367

This erratum concerns appendix B, where $A_{\epsilon}^{ \pm}$of equation (3.13) has been evaluated for $\epsilon \rightarrow 0$. The error is due to a too careless use of $F\left(-\frac{1}{4}, \frac{1}{2}, \frac{3}{4},\left(\frac{r_{\epsilon}^{ \pm}(\tau)}{r_{0}}\right)^{4}\right)=1+\mathcal{O}\left(\left(r_{\epsilon}^{ \pm}\right)^{4}\right)$ in the transition from equation (B.1) to (B.5). The argumentation would be clean if the integration over $\tau$ would be restricted to a finite interval. But in our case its two endpoints tend to $\pm \infty$, for $\epsilon \rightarrow 0$.

We now give the corrected result and sketch a new version of appendix B elsewhere.

Equations (B.7) and (3.13) have to be replaced by

$$
A_{\epsilon}^{ \pm}=\frac{\pi}{\epsilon(M \pm L / 2)}-\frac{8 \sqrt{2} \pi^{7 / 4} \sqrt{2 \sqrt{2}+3}}{\left(\Gamma\left(\frac{1}{8}\right)\right)^{2}} \frac{1}{\sqrt{\epsilon L}}+\mathcal{O}\left(\epsilon^{0}\right) .
$$

Consequently equations (3.16) and (4.3) have to be

$$
A_{\epsilon}=\frac{l_{1}+l_{2}}{\epsilon}-\frac{32 \pi^{7 / 4} \sqrt{2 \sqrt{2}+3}}{\left(\Gamma\left(\frac{1}{8}\right)\right)^{2}} \frac{1}{\sqrt{\epsilon\left|\vec{k}_{1}-\vec{k}_{2}\right|}}+\mathcal{O}\left(\epsilon^{0}\right)
$$

and

$$
\Omega(\lambda)=\frac{8 \pi^{3 / 4} \sqrt{2 \sqrt{2}+3}}{\left(\Gamma\left(\frac{1}{8}\right)\right)^{2}} \sqrt{\lambda}+\ldots
$$

respectively.

The numerical value of the new coefficient of the $1 / \sqrt{\epsilon}$-divergence differs from that of the old one by around two percent only. This explains, to some extent, why the error was not detected by an earlier rough numerical estimate of the $A_{\epsilon}^{ \pm}$integral. The numerics now fits the new asymptotic formulas perfectly.

Open Access. This article is distributed under the terms of the Creative Commons Attribution License (CC-BY 4.0), which permits any use, distribution and reproduction in any medium, provided the original author(s) and source are credited. 